LBNL-40495

June, 1997

\title{
Coherent Photons and Pomerons in Heavy Ion Collisions
}

\author{
Spencer Klein ${ }^{1}$ and Evan Scannapieco ${ }^{1,2}$ \\ ${ }^{1}$ Lawrence Berkeley National Laboratory, Berkeley, CA, 94720, USA \\ ${ }^{2}$ Department of Physics, University of California, Berkeley CA 94720-7304, USA.
}

\begin{abstract}
Ultrarelativistic heavy ion beams carry large electromagnetic and strong absorptive fields, allowing exploration of a variety of physics. $\gamma \gamma, \gamma P$, and $P P$ interactions can probe a huge variety of couplings and final states. RHIC will be the first heavy ion accelerator energetic enough to produce hadronic final states via coherent couplings. Virtual photons from the nuclear EM fields can interact in $\gamma \gamma$ interactions, which can be exploited to study many particle spectroscopy and QCD topics. Because the photon flux scales as $Z^{2}, \gamma \gamma$ luminosities are large up to an energy of about $\gamma \hbar c / R \approx 3 \mathrm{GeV} / \mathrm{c}$. Photon-Pomeron interactions are sensitive to how different vector mesons, including the $J / \psi$, interact with nuclear matter. $P P$ collisions rates are sensitive to the range of the Pomeron. Signals can be separated from backgrounds by using cuts on final state isolation (rapidity gaps) and $p_{\perp}$. We present Monte Carlo studies of different backgrounds, showing that representative signals can be extracted with good rates and signal to noise ratios.
\end{abstract}

(Presented at the 6th Conference on the Intersections of Particle and Nuclear Physics, May 27-June 2, 1997, Big Sky, Montana)

\section{PHYSICS PROCESSES}

When it is completed in 1999, the Relativistic Heavy Ion Collider [1] (RHIC) will be energetic enough to produce hadronic final states via $\gamma \gamma, \gamma P$, and $P P$ interactions that coherently couple to the nuclei as a whole. The electromagnetic field of the heavy nuclei can be considered as a flux, proportional to $Z^{2}$ of almostreal Weiszäcker-Williams virtual photons. The requirement that the photons couple to the entire nucleus limits the maximum photon energy to $\gamma \hbar c / R$, about $3 \mathrm{GeV} / \mathrm{c}$ for gold beams at RHIC. Thus, the maximum $\gamma \gamma$ energy is about $6 \mathrm{GeV}$; at energies of a few $\mathrm{GeV}$, the $\gamma \gamma$ luminosity will be comparable to those of the next generation $e^{+} e^{-}$colliders.

$\gamma \gamma$ interactions can probe a wide variety of physics topics. Particle coupling to two photons is a measure of their internal charge; $q \bar{q}$ mesons couple strongly, but glueballs and mixed states $(q \bar{q} g)$ should have much smaller couplings. Nonobservation in $\gamma \gamma$ collisions is therefore an important criteria for identification of glueball 

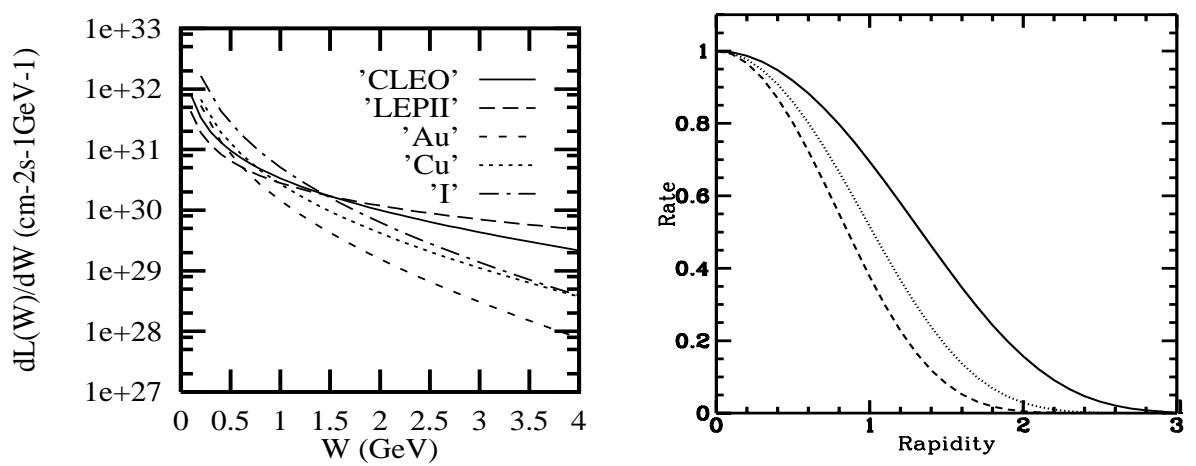

FIGURE 1. Left: Comparison of $\gamma \gamma$ luminosities at RHIC, for $\mathrm{Au}, \mathrm{I}$ and $\mathrm{Cu}$ beams, with those of CESR (CLEO) and LEP II. Right: Rapidity distribution of produced particles for gold on gold collisions with $\mathrm{W}=1$ (solid), 2 (dotted) and 3 (dashed) GeV.

candidates [2]. $\gamma \gamma$ collisions can also probe spin 0 or 2 mesons with exotic quantum numbers. One interesting example is the near-threshold $\rho^{0} \rho^{0}$ resonance, produced in $\gamma \gamma$ collisions at $e^{+} e^{-}$colliders. According to the particle data book, "This process has not been explained by models in which only conventional resonances dominate" [3].

$\gamma \gamma$ collisions also produce large numbers of lepton and hadron pairs. Lepton pairs can be used to measure the $\gamma \gamma$ and relative hadronic luminosity, and to search for nonlinear QED effects due to the large coupling $(Z \alpha \approx 0.6)$. Meson pairs can be used to measure form factors and for a variety of other QCD studies, and baryon pair production can test diquark based models [4].

Several authors have calculated the $\gamma \gamma$ luminosity produced by heavy ion colliders [5] [6] [7]. The $\gamma \gamma$ luminosity can be found by convoluting the photon fields of the two nuclei, and integrating over impact parameter $b$ greater than twice the nuclear radius $R_{A}$. The latter criteria avoids events where hadronic collisions overshadow the $\gamma \gamma$ interaction, but cuts the usable luminosity by about $50 \%$. Figure 1 compares the usable $\gamma \gamma$ luminosities for RHIC $\mathrm{Au}, \mathrm{Cu}$, and I beams to those of CLEO at CESR and LEP2. The lighter nuclei benefit from the higher $A A$ luminosity and beam energy, and smaller nuclear radius, which more than compensate for the reduced $Z$. Because of the photon energy cutoff, final states are produced quite centrally, as shown in Fig. 1. Final states from $e^{+} e^{-} \rightarrow \gamma \gamma$ have a wider $y$ distribution, and so, for a given setup, the experimental acceptance is lower.

Due to the nuclear form factor, the photons are almost real, with a maximum $Q^{2}$ given by the nuclear size, about $(30 \mathrm{MeV} / \mathrm{c})^{2}$ for gold. This cutoff limits the perpendicular momentum of the photons, $p_{\perp}<\hbar c / R$; this helps separate coherent from incoherent interactions. This is illustrated in Fig 2. The $Q^{2}$ limit will slightly reduce the rate of pair production near threshold [8].

In addition to $\gamma \gamma$ collisions, the virtual photons can also couple to the Pomeron field of the other nucleus. The Pomeron can be thought of as representing the absorptive part of the nuclear potential. $\gamma P$ interactions using proton targets were 
studied extensively at HERA. RHIC can study these interactions in a nuclear environment. One reaction of interest is $\gamma P \rightarrow V$, where $V$ is a vector meson. In the Vector Dominance Model, the photon can be considered to fluctuate into a spin 1 $q \bar{q}$ state, which then interacts with the nucleus. By studying production rates of various sized mesons, and varying the nuclear radius by changing the beams, the interactions between quark pairs and nuclei can be studied [9]. The kinematics are similar to $\gamma \gamma$ processes, so similar detection techniques can be used. RHIC will reach higher energies and luminosities than earlier NMC [10] and Fermilab E-665 [11] studies, producing 100,000's of exclusive $\rho$ and $\phi$ mesons per year, large numbers of excited states, and the $J / \psi$. The latter is of special interest because the large quark mass may require perturbative treatment.

In addition to photon physics, double Pomeron interactions can be studied. Unobscured $P P$ interactions can only occur in the impact parameter range $2 R_{A}+2 R_{P}>b>2 R_{A}$, where $R_{P}$ is the range of the Pomeron. The $P P$ cross section is thus sensitive to the range of the Pomeron. As $\gamma \gamma$ and $P P$ final states have similar kinematics, a statistical separation is required for this measurement. The relative rates will depend on $A ; P P$ couplings will dominate for small nuclei and $\gamma \gamma$ for large.

In many cases, the same final state can be produced through more than one intermediate state. Therefore, the possibility of interference exists. One place where it should occur is between the reactions $\gamma P \rightarrow V \rightarrow e^{+} e^{-}$and $\gamma \gamma \rightarrow e^{+} e^{-}$ [12]. The $\gamma \gamma$ and $\gamma P$ interactions are out of phase, so the interference angle comes from the real part of the Pomeron and phase shift of the vector meson in the nuclear potential.

\section{EXPERIMENTAL FEASIBILITY}

Measurements of coherent interactions are only possible if the signals can be separated from incoherent backgrounds [13]. This must be possible in both the final analysis and also at the trigger level; the latter appears to be the harder problem. The major backgrounds to be rejected are grazing nuclear collisions, photo-nuclear interactions, beam gas events, debris from upstream beam breakup, and cosmic ray muons; the latter two only affect triggering.

STAR (The Solenoidal Tracker at RHIC), is a general purpose large acceptance detector [14]. Time projection chambers track charged particles with pseudorapidity $|\eta|<2$ and $2.5<|\eta|<4$. A silicon vertex tracker, time of flight system and TPC $d E / d x$ help with particle identification. An electromagnetic calorimeter covers the range $-1<\eta<2$.

The coherent event trigger algorithms are based on requiring two or four tracks in the central TPC, with nothing else visible in the detector. Triggering uses a different set of detectors. The initial trigger selection uses scintillators and wire chambers (the anode wires in the TPC endcaps, with a fast readout) surrounding the TPC to select events based on multiplicity and topology. Two to five possible 


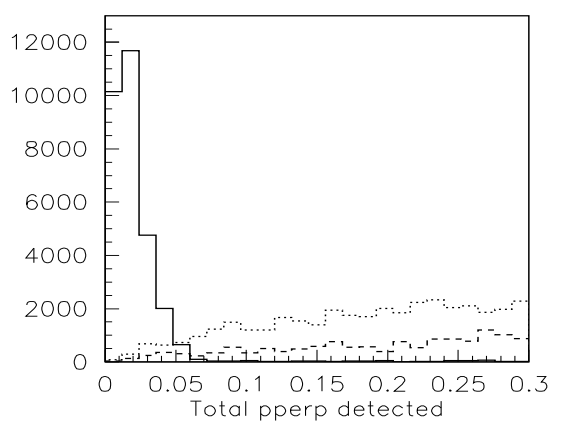

FIGURE 2. Comparison of $p_{\perp}$ between $\gamma \gamma$ and FRITIOF background events passing our cuts. The solid curve is for $\rho^{0} \rho^{0}$ production near threshold, the short dashes are beam-gas and the long dashes are peripheral nuclear backgrounds.

tracks are required, with a reasonable topology. Timing may be used to help reject cosmic ray muons and beam gas. Higher level trigger algorithms use better hit location information to select events with two or four charged tracks, with tighter topological cuts. Then, the calorimeter and TPC tracking contribute, allowing cuts on neutral multiplicity, total charge, vertex position, and $p_{\perp}$. Monte Carlo studies have shown that these trigger algorithms have good acceptance for coherent events, and adequate background rejection.

We have simulated the $\gamma \gamma$ signals and backgrounds from grazing nuclear and beam gas [15]. We generated tables of $\gamma \gamma$ luminosity as a function of invariant mass and rapidity, and then created events based on these tables. $p_{\perp}$ spectra are included with a $1 / R$ Gaussian form factor. Backgrounds were simulated with both the FRITIOF and Venus Monte Carlos; the $p_{\perp}$ spectra are compared in Fig. 2.

In order to study the effectiveness of different analysis techniques, we considered three sample analyses that are representative of a wide range of reactions; these analyses are listed in Table 1 . The $f_{2}(1270)$ is a well understood $q \bar{q}$ meson, representative of a wide class of particles that decay to two charged particles. A spin 0 particle with the same mass and decay channel would have a slightly lower acceptance. $\rho^{0} \rho^{0} \rightarrow \pi^{+} \pi^{-} \pi^{+} \pi^{-}$near threshold $\left(1.5 \mathrm{GeV}<M_{\rho \rho}<1.6 \mathrm{GeV}\right)$ is both a very interesting physics signal, and also a good benchmark for medium mass processes decaying to four charged particles. Finally, the $2960 \mathrm{MeV} J^{P C}=0^{-+}$ $c \bar{c}$ resonance $\eta_{c}$ was chosen as a real challenge; the luminosities are falling and the branching ratios are small.

For each analysis, we developed a set of cuts based on the required charged and neutral multiplicity and event topology. We required $p_{\perp}<100 \mathrm{MeV}$, and an appropriate invariant mass and found the rates and backgrounds given in Table 1. Although the FRITIOF and Venus background predictions are different, this analysis shows that $f_{2}(1270) \rightarrow \pi^{+} \pi^{-}$and threshold $\gamma \gamma \rightarrow \rho^{0} \rho^{0} \rightarrow \pi^{+} \pi^{-} \pi^{+} \pi^{-}$ reactions should be clearly separable from backgrounds, while the $\eta_{c} \rightarrow K^{* 0} K^{-} \pi^{+}$ may be accessible with particle identification.

We would like to thank our colleagues in the STAR collaboration. This work 
TABLE 1. Rates and backgrounds for $\gamma \gamma$ events for gold on gold collisions at RHIC for 3 sample analyses. The $\rho^{0} \rho^{0}$ events were near threshold, with invariant masses between 1.5 and $1.6 \mathrm{GeV} / \mathrm{c}^{2}$. The quantities in parenthesis assume particle identification by $d E / d x$ and TOF.

\begin{tabular}{|l|r|r|r|r|}
\hline Channel & Efficiency & $\begin{array}{r}\text { Detected } \\
\text { Events/Yr }\end{array}$ & $\begin{array}{r}\text { FRITIOF } \\
\text { Background }\end{array}$ & $\begin{array}{r}\text { Venus } \\
\text { Background }\end{array}$ \\
\hline$f_{2}(1270) \rightarrow \pi^{+} \pi^{-}$ & $85 \%$ & 920,000 & 53,000 & 100,000 \\
$\rho^{0} \rho^{0} \rightarrow \pi^{+} \pi^{-} \pi^{+} \pi^{-}$ & $38 \%$ & 16,000 & 3,500 & 1,400 \\
$\eta_{c} \rightarrow K^{* 0} K^{-} \pi^{+}$(w/ PID) & $57 \%$ & 70 & $210(8)$ & $510(20)$ \\
\hline
\end{tabular}

was supported by the DOE under contract DE-AC-03-76SF00098. E.S. has been partially supported by an NSF fellowship.

\section{REFERENCES}

1. Conceptual Design of the Relativistic Heavy Ion Collider, BNL-52195, May 1989, Brookhaven National Laboratory.

2. Paar, H., to appear in the Proceedings of Photon '97.

3. Particle Data Group, Barnett, R. M. et al., Phys. Rev. D54, 1 (1996); minireview on pg. 557-9.

4. Miller, D. J., in Lepton and Photon Interactions: XVI Intl. Symposium, Ithaca, 1993, ed. P. Drell and D. Rubin.

5. Cahn, R. N. and Jackson, J. D., Phys. Rev. D42, 3690 (1990).

6. Baur, G. and Ferreira Filho, F., Nucl. Phys. A518, 786 (1990).

7. Hencken, K., Trautmann, D., and Baur G., Z. Phys. C68, 473 (1995).

8. Baur, G. and Baron, N., Nucl. Phys. A561, 628 (1993).

9. Brodsky, S., Kinoshinta, T., and Terazawa, H., Phys. Rev. D4, 1532 (1971).

10. Ashman, J. et al., Z. Phys. C39, 169 (1988).

11. Baskin, B., these proceedings.

12. Leith, D. in Electromagnetic Interactions of Hadrons VI, ed. A. Donnachie and G. Shaw (Plenum Press, New York,1978).

13. Klein, S. R., in Photon 95, ed. D. J. Miller, S. L. Cartwright and V. Khoze (World Scientific, Singapore, 1996).

14. STAR Collaboration, STAR Conceptual Design Report, LBL-PUB-5347, 1992.

15. Klein, S. and Scannapieco, E., STAR Note 243, Feb. 1995, available at http://www.rsgi01.rhic.bnl.gov/star/starlib/doc/www/sno/ice/sn0243.html. 